\title{
Análisis de las inteligencias múltiples: un estudio con escolares de 11 a 13 años durante la pandemia sanitaria
}

\author{
Analysis of multiple intelligences: a study with 11- to 13-year-old \\ schoolchildren during the health pandemic
}

1 Esmeralda Giovanna Zapata Mocha (iD) https://orcid.org/0000-0002-7439-452X Universidad Técnica de Ambato Carrera de Pedagogía de la Actividad Física y Deporte. Tungurahua eg.zapata@uta.edu.ec

2 Julio Alfonso Mocha Bonilla (iD) https://orcid.org/0000-0002-8903-4746 Universidad Técnica de Ambato Carrera de Pedagogía de la Actividad Física y Deporte. Tungurahua ja.mocha@uta.edu.ec

3 Segundo Víctor Hernández del Salto (iD) https://orcid.org/0000-0002-9212-2747. Universidad Técnica de Ambato Carrera de Pedagogía de la Actividad Física y Deporte. Tungurahua, email vhernandez@uta.edu.ec

4 Judith del Carmen Núñez Ramírez (iD) https://orcid.org/0000-0001-8704-0563 Universidad Técnica de Ambato Carrera de Pedagogía de la Actividad Física y Deporte. Tungurahua judithdnunezr@uta.edu.ec

\section{Artículo de Investigación Científica y Tecnológica Enviado: 24/12/2021 \\ Revisado: $29 / 12 / 2021$ \\ Aceptado: 12/01/2022 \\ Publicado:08/03/2023 \\ DOI: https://doi.org/10.33262/concienciadigital.v6i1.4.2044}

Zapata Mocha, E. G., Mocha Bonilla, J. A., Hernández del Salto, S. V., \& Núñez Ramírez, J. del C. (2023). Análisis de las inteligencias múltiples: un estudio con escolares de 11 a 13 años durante la pandemia sanitaria. ConcienciaDigital, 6(1.4), 970-982. https://doi.org/10.33262/concienciadigital.v6i1.4.2044

CONCIENCIA DIGITAL, es una Revista Multidisciplinar, Trimestral, que se publicará en soporte electrónico tiene como misión contribuir a la formación de profesionales competentes con visión humanística y crítica que sean capaces de exponer sus resultados investigativos y científicos en la misma medida que se promueva mediante su intervención cambios positivos en la sociedad. https://concienciadigital.org

La revista es editada por la Editorial Ciencia Digital (Editorial de prestigio registrada en la Cámara Ecuatoriana de Libro con No de Afiliación 663) www.celibro.org.ec 


\section{Palabras} claves: inteligencias múltiples, desarrollo integral, inteligencias, educación, coeficiente intelectual.
Keywords: multiple intelligences, integral development, intelligences, education, iq.

\section{Resumen}

Introducción: La presente investigación se desarrolló durante el año 2021 en la etapa de confinamiento sanitario para conocer la importancia que tiene el desarrollo de las inteligencias múltiples en escolares de 11 a 13 años. Objetivo: Análisis las inteligencias múltiples en un grupo de escolares entre 11 a 13 años durante la pandemia sanitaria. Metodología: El estudio presenta una investigación de tipo cuantitativa, se utilizó un estudio descriptivo ya que se pudo observar y analizar los resultados de las inteligencias múltiples realizado con una muestra aleatoria aplicada a 40 estudiantes en tiempos de pandemia, para lo cual se utilizó el instrumento de IAMI para evaluar las inteligencias múltiples, la encuesta se aplicó de manera virtual para contribuir con las normas de bioseguridad sanitaria. Resultados: Los datos obtenidos se presentan en cuadros estadísticos y gráficos, contienen el análisis de: valores, medias y desviaciones estándares, así como las predominancias de las inteligencias múltiples. Conclusiones: Se determinó que las inteligencias múltiples que desarrollan los escolares en la presente muestra de estudio en el barrio Ingahurco se observa mayor preponderancia en la Inteligencia Intrapersonal seguido de la Inteligencia Kinestésica y Lingüística; en menor porcentaje tenemos la Inteligencia Lógico Matemático, la Inteligencia Interpersonal y la Musical, y con los porcentajes bajos tenemos a la Inteligencia Espacial Visual y la Naturalista.

\section{Abstract}

Introduction: The present research was developed during the year 2021 in the sanitary confinement stage in order to know the importance of the development of multiple intelligences in schoolchildren between 11 and 13 years old. Objective: Analysis of multiple intelligences in a group of school children between 11 and 13 years old during the sanitary pandemic. Methodology: The study presents a quantitative type of research, a descriptive study was used since it was possible to observe and analyze the results of multiple intelligences carried out with a random sample applied to 40 students in times of pandemic, for which the IAMI instrument was used to evaluate multiple intelligences, the survey was applied virtually to contribute with the sanitary biosecurity norms. Results: The data obtained are presented in statistical tables and graphs, they contain the analysis of: values, means and standard deviations, as 
well as the predominances of multiple intelligences. Conclusions: It was determined that the multiple intelligences developed by the schoolchildren in the present study sample in the Ingahurco neighborhood show a greater preponderance of Intrapersonal Intelligence followed by Kinesthetic and Linguistic Intelligence; in lower percentages we have Logical Mathematical Intelligence, Interpersonal Intelligence and Musical Intelligence, and with low percentages we have Visual Spatial Intelligence and Naturalistic Intelligence.

\section{Introducción}

El campo laboral de la pedagogía de la actividad física y deporte, se lo realiza de acuerdo al Ministerio de Educación en la educación física en unidades educativas, escuela y colegios públicos y privados, los nuevos profesionales en el ámbito deportivo se cuestionan cuáles son las metodologías apropiadas para que los estudiantes desarrollen competencias de forma autónoma y eficaz (Aldana, 2016). Los docentes de educación Física toman en cuenta el desarrollo cognitivo que están acompañados por aspectos sociales y emocionales, que funcionan de manera instantánea, el conocimiento el contexto educativo y la instrucción, a esto se denomina aplicación de las inteligencias múltiples (Garner, 2001).

El proceso de enseñanza- aprendizaje está relacionado directamente en la forma como los seres humanos aprenden (Guzmán, 2017), y este aprendizaje está relacionado con los estímulos cerebrales Armstrong (2006) que fortalecen el aprendizaje de forma positiva o negativa, dependerá también de los conocimientos previos, las emociones las situaciones (González, 2013), y que fortalecerán el desarrollo físico la atención, y las inteligencias (Hunt, 2004). En este proceso de aprendizaje se requiere la participación activa de los estudiantes (Ullauri, 2019), que tienen aptitudes y competencias (Garner, 1995) e inteligencias integrales que las evidencias a través del cumplimiento de las instrucciones y el desarrollo del ejercicio.

El docente de la actividad física y deporte debe dominar el comportamiento de las inteligencias múltiples en los estudiantes (Galán, 2001), para que las instrucciones se conviertan en herramientas de comunicación efectiva entre el conocimiento y la actividad (Cazau, 2006), lo cual fortalecerá la interrelación entre el docente y el estudiante (Fernández \& Ruiz, 2007), sin dejar de lado la importancia que tiene el apoyo motivacional del docente hacia la construcción de valores cognitivos y socio afectivos durante la labor pedagógica (Bonilla et al., 2020), lo cual le permita identificar por cuál 
de las inteligencias múltiples puede cumplir los objetivos propuestos en la planificación curricular (Gallego et al., 2021).

Si la identificación de las inteligencias múltiples fortalecerá el proceso de enseñanza aprendizaje (Macías et al., 2021), es importante acércanos a una conceptualización desde la práctica de la educación física (Espinosa et al., 2021), puesto que es ahí donde los estudiantes exteriorizan: emociones, sentimientos, aspiraciones, conocimientos, preferencias por disciplinas deportivas, cuidado de su cuerpo y desarrollo del mismo y sobre todo saber escuchar de forma activa las instrucciones de los facilitadores en cuanto al nuevo conocimiento (Góngora et al., 2008).

Los seres humanos en el proceso de aprendizaje tienen características propias que están relacionadas directamente al gran espectro de inteligencias (Pacheco et al., 2021). La teoría de las inteligencias múltiples establece que cada sujeto posee un perfil característico de inteligencias. Por ello es necesario conocer los rasgos de nuestros alumnos para poder determinar el principio de intervención didáctica; las inteligencias múltiples definidas por Garner (1995) como: "la capacidad de resolver problemas o crear productos que sean valiosos en uno o más ambientes culturales“ (pág.10), implica que el docente debe tomar en cuenta para su ejercicio profesional el contexto cultural, las experiencias recibidas de los estudiantes como un preámbulo para poder solucionar situaciones de aprendizaje, aspectos de interrelación social y sobre todo que el conocimiento esté orientado al desarrollo de destrezas, según el género (González, 2013) la excelencias de estás permitirá la mejora de competencias.

En tal sentido Espinosa et al. (2021), menciona que existe un espectro de habilidades humanas, las cuales, al ponerlas en práctica, las personas no solo se sentirían mejor sobre sí mismas, sino que serían más competentes en el entorno social, se sentirán más comprometidas y habilitadas para unirse con el resto de la comunidad y hacer un mundo mejor. A su vez Altarejos (2004), menciona a la inteligencia como: "la habilidad cognitiva para poder manejar las demandas de trabajo y conducta es instrumento al servicio de la vida" (p.116). por medio de esta definición relaciona a la inteligencia y a la educación de una manera directa (Paoloni et al., 2014), y que los estudiantes pueden beneficiarse de manera personal y en equipo al practicar alguna disciplina deportiva del proceso educativo e inter aprendizaje. Otros actores le definen a la inteligencia como mecanismos procesuales que nos ayudan a: decodificar información, analizar y razonar sobre los conflictos y metas que los estudiantes deben sobre llevar en el aprendizaje cotidiano, Suárez \& Barrios (2012), manifiesta que: “es el proceso por el cual percibimos información por medio de conexiones secuenciales destacando las razones lógicas, la causa y el efecto" (p.5). Por este motivo se identifican que cuando los seres humanos podemos interpretar aspectos cotidianos con más o menos inteligencia poseen un determinado coeficiente intelectual. 
Según González (2013) en su investigación de psicomotricidad aplicada como actividad corporal, se considera una influencia directa en la estimulación de las inteligencias múltiples en los estudiantes, se observa en su investigación que la práctica de diferentes actividades psicomotores influye en la estimulación de diversas áreas llamadas inteligencias múltiples. Por su parte Cruz \& Cruzata (2017), en un estudio sobre inteligencia emocional y kinestésica de la educación física primaria, afirma que la inteligencia kinestésica tiene características observables, se encarga de controlar los movimientos, el equilibrio, la velocidad, la coordinación y la flexibilidad; las inteligencias múltiples no solo se enfoca en la parte deportiva sino también en la educación física considerándola como lenguaje corporal (Ullauri, 2019), pero también se produce el fortalecimiento del aprendizaje y el desarrollo de la parte social ya afectiva (Torres-Silva \& Díaz-Ferrer, 2021).

Las inteligencias múltiples de la teoría de Garner (1995), proporcionan información a los futuros docentes sobre el desarrollo de habilidades sociales, de liderazgo y cooperación, resolución de conflictos, con inteligencia y de manera equilibrada a través de la metodología que apliquen para que exista un aprendizaje significativo de los conocimientos (Zárate \& Reina, 2020). La inteligencias musicales adquiere la capacidad que tenemos los seres humanos: percibir discriminar, transformar y expresar aspectos musicales, las personas aportan con la sensibilidad que tienen al ritmo, el tono y timbre, los estudiantes se sienten a traídos por edificar sonidos de la naturaleza y por cualquier tipo de manifestación sonora que pueden expreses arló con silba, cantar o seguir el compas con el pie, golpeando o sacudiendo algún objeto que tengan a la mano y que sirva para emitir sonidos (Armstrong, 2006). Mientras que la Inteligencia corporal-cinestésica es la que más desarrolla los jóvenes por que utilizan el cuerpo como medio de expresión de ideas, sentimientos, emociones por medio de la cual procesan el conocimiento y reflejan en la expresión gesticular, coordinación, destreza, equilibrio, flexibilidad, saltabilidad, fuerza con el cuerpo y estas capacidades les permite destacarse en el desarrollo de disciplinas deportivas, danza y cualquier expresión corporal que ellos ejecutan (Armstrong, 2006), así la inteligencia lingüística es una de las capacidades del ser humano es la comunicación de ahí que el uso de la palabra sea de forma oral o escrita incluye una habilidad para el uso adecuado de la sintaxis, semántica, fonética, y los usos pragmáticos del lenguaje en la vida codiana, nos sirve para hablar correctamente, escuchar de forma activa, leer y decodificar la información que está en el entorno (Armstrong, 2006). A su vez la inteligencia lógico-matemática es una capacidad para utilizar de forma efectiva y razonar adecuadamente el uso de los números por medio de ecuaciones, relaciones lógicas, afirmaciones, proposiciones, funciones, abstracciones y problemas planteados que requieren de cálculos numéricos y estadísticos, puesto que los números tiene lógica, patrones y secuencias que estimulan el razonamiento lógico de los estudiantes (Gardner, 1995). Por otra parte, la inteligencia espacial permite al ser humano en tres dimensiones, percibir imágenes externas e internas, recrearlas transformarlas y 
presentarlas de manera gráfica, en esquemas o cuadros en el ámbito educativo son los mapas conceptuales y mentales, con los croquis o planos que utilizan ilustraciones para expresar las ideas (Armstrong, 2006). La Inteligencia interpersonal permite que las personas se relacionen y se entiendan con otras similares, permite una interacción efectiva con una o más personas, amigos, familiares, compañeros y público en general que por medio de la comunicación se exterioriza la sensibilidad, los sentimientos, estados de ánimo que ayuden a una comunicación afectiva y efectiva Gardner (2001), La inteligencia intrapersonal es aquella cuando los seres humanos desarrollamos la habilidad para reconocer quienes somos y cuáles son las capacidades y competencias que tenemos para ejecutar una acción, también permite desarrollar la conciencia sobre las fortalezas y debilidades que poseemos que nos ayuden a cumplir metas u objetivos que nos trazamos a lo largo de la vida, finalmente la Inteligencia naturalista es la capacidad para distinguir, clasificar utilizar los elementos del medio ambiente con una alta conciencia de protección, observación y reflexión sobre el beneficio o daño que hacen las acciones en contra o a favor de la naturaleza, ese sentimiento de protección por animales, plantas e investigar las características del mundo natural permite que el ser humano pueda vivir en armonía con el mundo en el que vive (Gardner, 2001). En este sentido luego de haber establecido cuales son los tipos de inteligencias, el estudio fue realizado con el propósito de analizar las inteligencias múltiples de una muestra de escolares entre 11 a 13 años de edad durante la etapa de la pandemia del COVID - 19.

\section{Metodologia}

El corte del estudio presenta una investigación de tipo cuantitativa, se utilizó esta modalidad de investigación porque se recogió, tabuló y analizó datos numéricos relacionados con la muestra de estudio, el alcance fue descriptivo ya que se pudo observar y analizar los resultados obtenidos de las inteligencias múltiples. El trabajo investigativo por motivos de la pandemia del COVID-19 fue de tipo descriptivo según lo señala Posso et al. (2021), en un estudio descriptivo se analizan una serie de cuestiones, conceptos y variables con el fin de describirlas; al mismo tiempo se puede medir el grado de relación entre 2 o más variables, por consiguiente, se buscó analizar los tipos de inteligencias múltiples que presentan los escolares del sector Ingahurco de la ciudad de Ambato.

Sujetos. La muestra de estudio fue conformada por 40 estudiantes en edad escolar de 11 a 13 años de varias Unidades Educativas del cantón Ambato, cabe destacar que por motivos de la pandemia la muestra es pequeña, la cual se tomó de un sector urbano, del Nor centro de la ciudad de Ambato, específicamente del barrio Ingahurco, por razones de seguridad en la salud de los escolares las encuestas fueron realizadas durante el año 2021 de manera virtual para cumplir con las disposiciones emitidas por el COE nacional y provincial, que actuaron en relación directa con el Ministerio de Educación y los distritos Zonales para precautelar la salud de estudiantes, docente y directivos que acuden a las 
instituciones de educación y que recién en el 2021 se dio inicio a la vacunación máxima en el Ecuador.

Instrumento. Para conocer los tipos de inteligencias que se presentan en la muestra de estudio, se aplicó el Inventario de Autoeficacia para las Inteligencias Múltiples cuyas siglas son (IAMI), es decir se estableció un cuestionario estructurado, que se lo aplicó de manera virtual.

Protocolo. Por motivos de la pandemia del covid-19 y la emergencia sanitaria del año 2021 presente en la ciudad de Ambato, se buscó alternativas para realizar el estudio, en primera instancia basados en las clases virtuales de los escolares de las instituciones educativas del sector de Ingahurco, se mantuvo contacto vía telefónica con los padres de familia para solicitar el consentimiento y proceder a enviar el inventario (IAMI), establecido como una encuesta virtual realizada en un formulario online, con lo cual el enlace se envió a los padres y representantes de los escolares, se realizó una reunión virtual para informar del objetivo del estudio, los padres conjuntamente con sus hijos procedieron a llenar el formulario, todas las respuesta fueron registradas por el grupo de investigadores para consolidarlas y analizarlas, el objetivo fue analizar las inteligencias múltiples que presentan los escolares de 11 a 13 años de las instituciones educativas del sector Ingahurco.

\section{Resultados}

Caracterización de la muestra de estudio

La primera actividad fue el análisis y presentación de los resultados de la presente investigación, se realizó el proceso de caracterización de la muestra de estudio, en base a las variables recolectadas para ello (tabla 1 ).

Tabla 1

Caracterización de la muestra de estudio

\begin{tabular}{cccccccccccc}
\hline \multirow{2}{*}{ Sexo } & f & \multirow{2}{*}{$\%$} & \multicolumn{3}{c}{ Edad (años) } & \multicolumn{3}{c}{ Estatura $(\mathrm{cm})$} & \multicolumn{3}{c}{ Peso $(\mathrm{kg})$} \\
\cline { 4 - 13 } & & & M & DS & P & M & DS & P & M & DS & P \\
\hline Femenino & 22 & 55.0 & 12.0 & 0.7 & & 143.6 & 5.2 & & 37.5 & 5.4 & \\
Masculino & 18 & 45.0 & 11.9 & 0.7 & $0.748^{*}$ & 147.1 & 5.8 & $0.050^{* *}$ & 47.0 & 8.8 & $0.000^{* *}$ \\
Total & 40 & 100.0 & 11.9 & 0.7 & & 145.1 & 5.7 & & 41.7 & 8.5 & \\
\hline
\end{tabular}

En la tabla 1 se puede observar el análisis de las frecuencias (f) y porcentajes (\%) por grupos de género, con determinación de valores medios $(\mathrm{M})$ y desviaciones estándares (DS) de la variable de la edad, estatura y peso con diferencias significativas en un nivel de $\mathrm{P}<0.05(* *)$ y $\mathrm{P} \geq 0.05\left(^{*}\right)$ entre grupos por género. La tabla refleja las diferencias significativas en edad, estatura y peso entre el sexo masculino y femenino, luego se 
compararon los resultados de (M) en edad, tienen el mismo valor medio en estatura, el grupo masculino tiene un aumento de desviación estándar mayor que al grupo femenino y en peso el grupo de sexo femenino tiene una baja desviación estándar.

En base al instrumento de investigación utilizado para la valoración de las inteligencias múltiples, se determinó la valoración de cada una de las inteligencias (tabla 2) y la predominancia porcentual en la muestra de estudio (figura 1).

\section{Tabla 2}

Valoración de las inteligencias múltiples en la muestra de estudio.

\begin{tabular}{cccccc}
\hline Inteligencias múltiples & $\mathrm{N}$ & Mín & Máx & $\mathrm{M}$ & DS \\
\hline Lingüística & 40 & 3.60 & 9.80 & 7.6100 & 1.72163 \\
Lógico matemática & 40 & 2.20 & 9.60 & 6.5650 & 1.64979 \\
Espacial visual & 40 & 3.00 & 8.40 & 6.2300 & 1.36817 \\
Musical & 40 & 1.20 & 10.00 & 6.0750 & 2.08520 \\
Interpersonal & 40 & 2.80 & 10.00 & 7.0500 & 1.75572 \\
Kinestésico corporal & 40 & 3.200 & 10.000 & 7.12500 & 2.217732 \\
Intrapersonal & 40 & 4.000 & 10.000 & 7.72500 & 1.921371 \\
Naturalista & 40 & 1.20 & 9.40 & 6.5900 & 1.82684 \\
\hline
\end{tabular}

El análisis evidencia los valores mínimos (Mín), máximos (Máx), medios (M) y desviaciones estándares (DS) de la valoración de las inteligencias múltiples.

Tabla 3

Las inteligencias múltiples por género

\begin{tabular}{|c|c|c|c|c|c|c|c|}
\hline \multirow{2}{*}{$\begin{array}{c}\text { Inteligencias } \\
\text { múltiples }\end{array}$} & \multicolumn{3}{|c|}{ Masculino } & \multicolumn{3}{|c|}{ Femenino } & \multirow{2}{*}{$\mathrm{P}$} \\
\hline & $\mathrm{n}$ & $M$ & DS & $\mathrm{n}$ & $\mathrm{M}$ & DS & \\
\hline Lingüística & 22 & 7.4000 & 1.57117 & 18 & 7.8667 & 1.90356 & $0.205^{*}$ \\
\hline Lógico matemática & 22 & 6.7909 & 1.11863 & 18 & 6.2889 & 2.13346 & $0.723 *$ \\
\hline Espacial visual & 22 & 6.2364 & 1.29561 & 18 & 6.2222 & 1.49019 & $0.870 *$ \\
\hline Musical & 22 & 6.4091 & 1.84698 & 18 & 5.6667 & 2.33238 & $0.531 *$ \\
\hline Interpersonal & 22 & 7.4545 & 1.58707 & 18 & 6.5556 & 1.86807 & $0.142^{*}$ \\
\hline
\end{tabular}


Tabla 3

Las inteligencias múltiples por género (continuación)

\begin{tabular}{cccccccc}
\hline $\begin{array}{c}\text { Inteligencias } \\
\text { múltiples }\end{array}$ & \multicolumn{3}{c}{ Masculino } & \multicolumn{3}{c}{ Femenino } & P \\
\hline Kinestésico & 22 & 6.96364 & 2.059715 & 18 & 7.32222 & 2.442890 & $0.445^{*}$ \\
& & & & & & & \\
Intrapersonal & 22 & 7.86364 & 1.588514 & 18 & 7.55556 & 2.301463 & $0.902^{*}$ \\
Naturalista & 22 & 6.4818 & 1.99777 & 18 & 6.7222 & 1.64086 & $0.870^{*}$ \\
\hline
\end{tabular}

Los análisis descriptivos reflejan los valores medio (M) y desviaciones estándares (DS) sin diferencias significativas en un nivel de $\mathrm{P} \geq 0.05$ entre grupos por género. Tal y como se observan en los resultados en la tabla 3 se agrupan la Inteligencias múltiples en alta: Inteligencia Intrapersonal, Inteligencia lingüística y la Inteligencia Kinestésico; la media con resultados similares tenemos: Inteligencia interpersonal, Inteligencia lógico matemático, musical, para finalizar, las inteligencias más bajas son: Naturalista y Espacial y Visual.

\section{Figura 1}

Analisis de las intelgenecias multiples

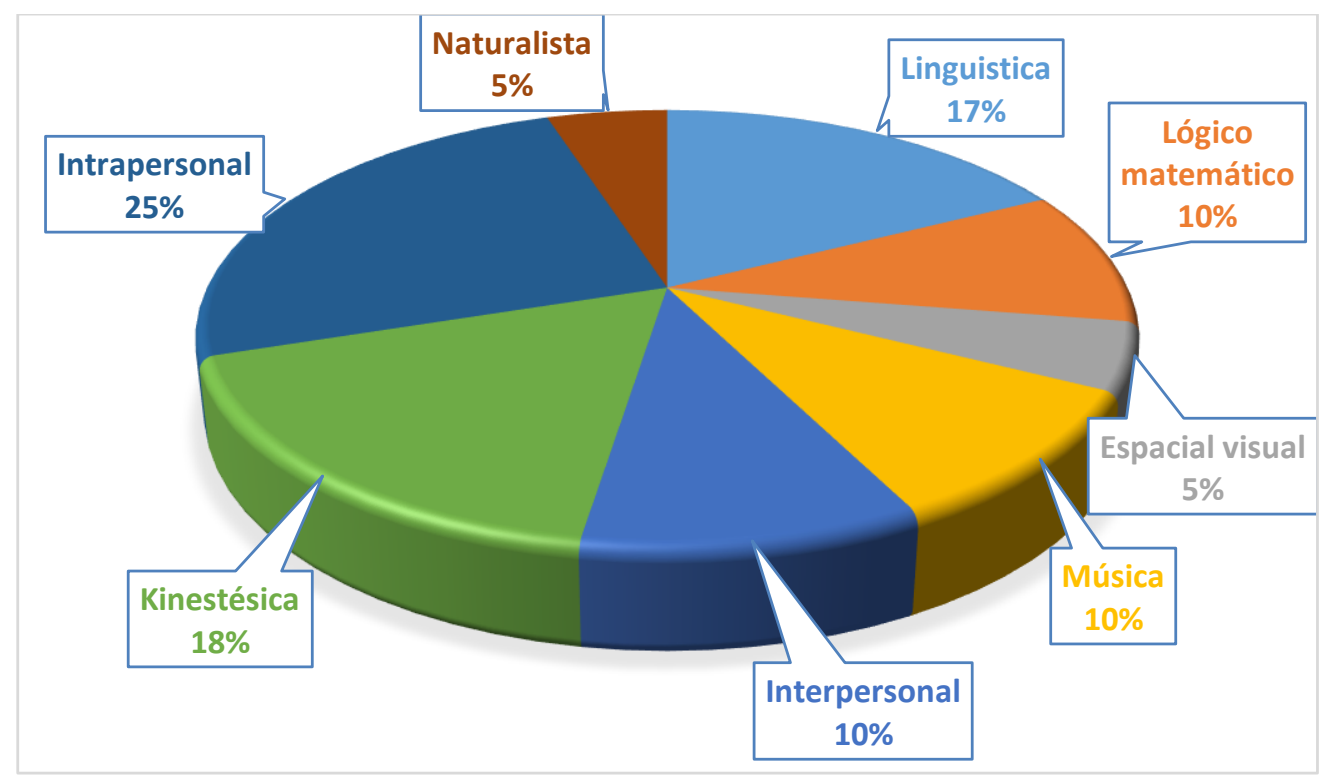


En los resultados también podemos observar claramente que el mayor porcentaje que tiene desarrollado los niños es la de Inteligencia Intrapersonal, seguido de la Inteligencia Kinestésica, luego tenemos la Inteligencia Lingüística; en menor porcentaje tenemos la Inteligencia Lógico Matemático y la Inteligencia Interpersonal y la Musical; finalizando con los porcentajes bajos tenemos a la Inteligencia Espacial Visual y la Naturalista.

\section{Conclusiones}

- Según Cruz \& Cruzata (2017), en su investigación titulada inteligencia emocional y kinestésica en la educacion física se concluye que el $10.83 \%$ de estudiantes se encuentran en un bajo nivel de desarrollo, en lo que corresponde a la inteligencia emocional llamado también intrapersonal, sin embargo, en nuestro estudio se pudo evidenciar que un 25,00\% de los estudiantes se encuentran con un alto nivel de desarrollo de la inteligencia intrapersonal. Por otra parte, en relación con la inteligencia Kinestésica corporal el $38.76 \%$ de los estudiantes alcanzaron un nivel bueno, a comparación con los resultados de nuestro estudio donde se pudo observar un promedio alto de $18.00 \%$ en definitiva un nivel muy bueno.

- Los resultados de este trabajo investigativo resaltan el desarrollo de la inteligencia intrapersonal en jóvenes entre 11 y 13 años, tanto hombres como mujeres, que están en un proceso de crecimiento corporal e intelectual y que en el día día del proceso educativo fortalecerán las capacidades para potenciarlas en competencias, por lo que el rol de los docentes y del proceso educativo es fortalecer el aprendizaje significativo y el desarrollo optimo de la inteligencia intrapersonal.

\section{Referencias bibliográficas}

Aldana, S., Vereda, F., Hidalgo-Alvarez, R., \& de Vicente, J. (2016). Facile synthesis of magnetic agarose microfibers by directed selfass.

Altarejos, F. (2004). Autorregulación e integración: dos propuestas en la educación de la afectividad (D. Goleman y Tomás de Aquino). Estudios sobre educación, 7, 4566.

Armstrong, T. (2006). Inteligencias múltiples en el aula. Guía práctica para educadores. Barcelona, Paidós.

Bonilla, J. A. M., Benítez, M. C., \& Galak, E. L. (2020). Apoyo motivacional docente y necesidades psicológicas básicas en la Educación Física: una revisión sistemática. Ciencia Digital, 4(1.1.), 5-20. 
Cazau, P. (2006). investigación exploratoria, descriptiva, correlacional y explicativa. Introducción a la investigación en ciencias sociales, 25-32.

Cruz Ordoñez, A. D. L., \& Cruzata Martínez, A. (2017). Inteligencia emocional y kinestésica en la educación física de la educación primaria. Actualidades Investigativas en Educación, 17(2), 262-281.

Espinosa, Y., Arcia, F. M., \& González, P. F. (2021). Los estilos de aprendizaje y las inteligencias múltiples en estudiantes del colegio Francisco de Paula Santander. Revista de Estilos de Aprendizaje, 14(28), 234-247.

Fernández, N. G., \& Ruiz, M. G. (2007). El Aprendizaje Cooperativo como estrategia de Enseñanza-Aprendizaje en Psicopedagogía (UC): repercusiones y valoraciones de los estudiantes. Revista Iberoamericana de Educación, 42(6), 1-13.

Galán Guerrero, L.P. (2021). Estrategias didácticas para fortalecer inteligencias múltiples en niños 4-6 años en el hogar Comunitario Infantil Cebollitas Ocaña Norte de Santander.

Gallego, A.G., Pérez, A.M., Fernández, V.F., Alcántara-López, M., y Sáez, M.C. (2021). Satisfacción con la vida en adolescentes: relación con el estilo parental, el apego con los compañeros y la inteligencia emocional. Revista electrónica de investigación en psicología de la educación, 19 (53), 51-74.

Gardner, H. (1995). Inteligencias múltiples. La teoría en la práctica. Barcelona, Paidós.

Gardner, H. (2001). La inteligencia reformulada: las inteligencias múltiples en el siglo XXI (No. 159.955 G171i Ej. 1 020338). Paidos.

Góngora, D. P., Cortés, M. D. C. M., Morón, M. T. P., Martín, C. R. R., \& Martínez, F. M. (2008). La competencia lingüística como base del aprendizaje. International Journal of Developmental and Educational Psychology, 2(1), 177-183.

González Cubillan, L. \&. (2013). Geográfica, estimación de la inteligencia interpersonal e intrapersonal según el género y la ubicación. Psicogente, 16(30), 368-378. https://www.redalyc.org/articulo.oa?id=497552364010.

Guzmán, B. (2017). Las inteligencias múltiples en el aula de clases. Revistas de Investigación, 29. http://revistas.upel.edu.ve/index.php/revinvest/article/view/4114/2085

Hunt, E. (2004). Multiple views of multiples intelligence [Recension de intelligence reframed: multiples intelligence in the 21 st century]. Contemporary Psychology, 46(1). 
Macías Figueroa, Y. G., Vigueras Moreno, J. A., \& Rodríguez Gámez, M. (2021). Una escuela con inteligencias múltiples: visión hacia una propuesta innovadora. Revista Cubana de Educación Superior, 40(1).

Pacheco, R. J. P., Arias, S. P. V., Ñacato, J. C. M., Toapanta, P. F. C., \& Pérez, N. E. M. (2021). Inteligencias múltiples como estrategia para la Educación Física: una intervención didáctica durante la pandemia. PODIUM-Revista de Ciencia y Tecnología en la Cultura Física, 17(1).

Paoloni, P.V., Rinaudo, M.C. \& González Fernández, A. (2014). El papel de las emociones en los aprendizajes académicos. Cuestiones en Psicología Educacional: perspectivas teóricas, metodológicas y estudios de campo. Tenerife: Sociedad Latinoamericana de Comunicación Social (SLCS).

Posso Pacheco, R. J., Otáñez Enríquez, N. R., Cóndor Chicaiza, J. D. R., Cóndor Chicaiza, M. G., \& Lara Chala, L. D. R. (2021). Educación Física remota: juegos motrices e inteligencia kinestésica durante la pandemia COVID-19. Podium. Revista de Ciencia y Tecnología en la Cultura Física, 16(2), 564-575.

Suárez, C. S., \& Barrios, L. (2012). El cerebro triuno y la inteligencia ética: matriz fundamental de la inteligencia multifocal. Praxis, 8(1), 147-165.

Torres-Silva, L., \& Díaz-Ferrer, J. (2021). Inteligencias múltiples en el fortalecimiento del aprendizaje cooperativo efectivo. IPSA Scientia, revista científica multidisciplinaria, 6(1), 64-80.

Ullauri Ortiz, M. J. (2019). Análisis de la inteligencia kinestésica corporal y la coordinación motriz en bachilleres (Bachelor's thesis, Quito: UCE).

Zárate, J. J. M., \& Reyna, C. D. C. E. (2020). La teoría de las inteligencias múltiples y su implicación en la enseñanza de la historia. REIDICS. Revista de Investigación en Didáctica de las Ciencias Sociales, (6).

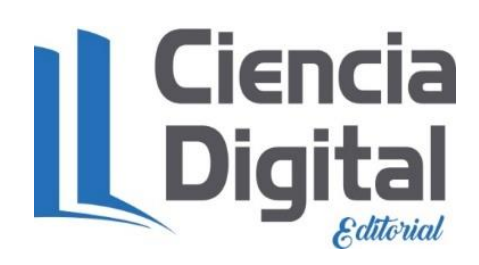


El artículo que se publica es de exclusiva responsabilidad de los autores y no necesariamente reflejan el pensamiento de la Revista Conciencia Digital.

\section{Liencia}

El artículo queda en propiedad de la revista y, por tanto, su publicación parcial y/o total en otro medio tiene que ser autorizado por el director de la Revista Conciencia Digital.
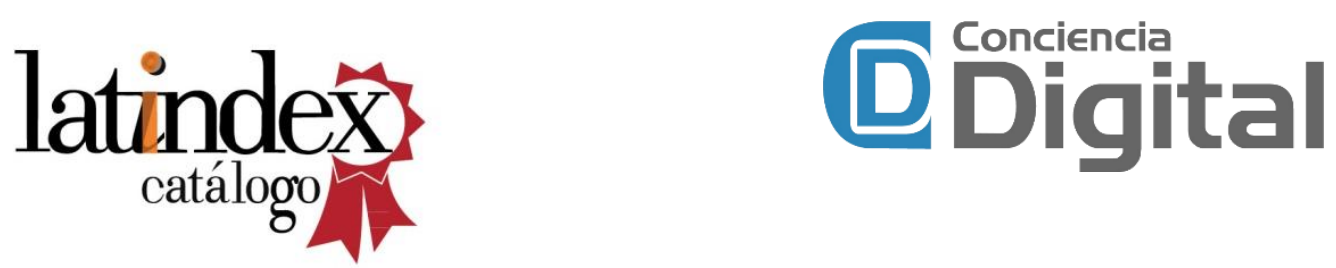

Indexaciones

doi crossref latindex̃

